\section{Gli esami non mentono in CKD-MBD: PTH, Vitamina D \& carbamazepina}

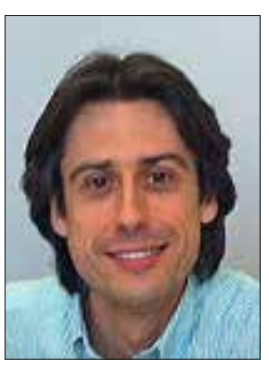

Andrea Galassi un iter diagnostico di impronta olistica. La carenza di $25(\mathrm{OH})$ $D$ e l'effetto della carbamazepina sulle reazioni di attivazione e degradazione della Vitamina D, mediate dai citocromi P450 in corso di IRC, sono presentati come i principali responsabili della resistenza al paracalcitolo. Vorrei soffermarmi su tre momenti della storia clinica, che rendono questo caso di portata emblematica nella terapia della sindrome.

\section{Fase iniziale}

Quando nel 2006 il paziente di 37 anni giungeva all'attenzione del Nefrologo, presentava già IRC avanzata in stadio 5, con grave IPS, associato a ipocalcemia e fosforemia borderline. La storia clinica dimostra come questi insoliti livelli di PTH meritassero sin da allora particolare attenzione. Benché l'articolo non descriva precedenti terapie della CKDMBD, anche considerando il paziente un late referral, PTH così elevati sarebbero stati solo parzialmente giustificati dall'esposizione a un protratto sovraccarico di fosforo (solo in parte rappresentato dalla fosforemia), da un bilancio calcico negativo e dalla carenza di Vitamina D. È ragionevole pensare che la gravità dell'IPS esprimesse da subito una spiccata inattività della Vitamina $D$ endogena mediata dalla carbamazepina.

Benché la correzione della carenza di 25(OH)D costituisca il primo step nel trattamento dell'IPS, è noto come i livelli di PTH vengano ridotti dalla sola terapia con Vitamina $D$ nativa molto meno di quanto non avvenga in corso di terapia con le sue forme attive, specialmente in dialisi $(4,5)$. È pertanto discutibile che in questa fase il colecalciferolo, anche a dosi elevate, avrebbe corretto i livelli di PTH, considerando non solo l'effetto positivo sulla conversione del colecalciferolo in 25(OH)D ma anche la degradazione di quest'ultimo, indotti dalla carbamazepina. Del resto anche tra il 2011 e il 2014, in corso di colecalciferolo a dosi carico, i livelli di 25(OH)D non superavano il target di $30 \mathrm{ng} / \mathrm{mL}$. Credo vi sia piuttosto un altro elemento saliente: se già nel 2006 fosse stata correttamente posta diagnosi di carenza di $25(\mathrm{OH}) \mathrm{D}$, la sua difficile correzione avrebbe fornito un ulteriore elemento diagnostico per indurre più precocemente il sospetto di una "tossicità" minerale indotta dalla carbamazepina.

\section{Fase dialitica}

L'andamento clinico tra il 2007 e il 2011 rappresenta un autentico paradigma di resistenza alla Vitamina $D$ attiva. I livelli di PTH, calcemia e fosforemia non sembrano sconvolgenti: molti dializzati presentano PTH di $600-700$ pg/mL con fosforemie moderatamente elevate. Nessun dato di laboratorio dovrebbe essere però interpretato senza considerare le terapie. Già nel 2007, la dose di paracalcitolo (30 mcg/sett.) era adeguata per massimi livelli di iPTH intorno a $700 \mathrm{pg} / \mathrm{mL}$ (considerando l'indicazione della scheda tecnica a determinare la dose iniziale dividendo i valori basali di PTH per 80: 700/80 = 8.75 mcg ev 3 volte alla settimana). II successivo aumento del PTH verso $970 \mathrm{pg} / \mathrm{mL}$ sotto paracalcitolo a dosi congrue con un'incompleta risposta clinica (PTH sempre intorno a $500 \mathrm{pg} / \mathrm{mL}$ ), nonostante l'aumento delle dosi a $45 \mathrm{mcg} / \mathrm{sett}$. nel 2009 (superiori alle raccomandazioni: 970/80 = $12 \mathrm{mcg}$ x 3/sett.), descrive un caratteristico caso di "resistenza" al paracalcitolo.

Ancora più insoliti sono i livelli di calcemia ai limiti inferiori in corso di paracalcitolo a dosi tanto elevate. I casi più comuni di resistenza alla Vitamina D attiva si associano alla down-regulation dei VDR nell'iperparatiroidismo terziario con ipercalcemia. L'assetto metabolico del paziente suggeriva diversamente un'anomalia non ascrivibile al paracalcitolo di per sé o all'evoluzione terziaria, bensì a un accelerato catabolismo del paracalcitolo indotto dalla carbamazepina. Si potrebbe dire che i VDR del paziente ricevessero una dose irrisoria di paracalcitolo rispetto a quella somministrata.

\section{Paratiroidectomia e trapianto}

La persistenza di PTH elevati dopo paratiroidectomia 7/8 indurrebbe il sospetto di un adenoma residuo, ma, ancora una volta, la normocalcemia e, soprattutto, l'andamento favorevole del PTH nel post-trapianto, ad avvenuta sospensione di calciomimetico e paracalcitolo, scongiurano la presenza di forme terziarie rimandando alla resistenza alla Vitamina D.

Tuttavia, credo occorra maggiore prudenza nel soppesare gli effetti del colecalciferolo. Il dimezzamento del PTH è stato, infatti, concomitante non solo all'introduzione del colecalciferolo, ma anche alla paratiroidectomia, all'associazione del calciomimetico e alla ripresa di funzione del trapianto. Sembra qui impossibile discriminare quale degli interventi abbia fatto singolarmente la differenza. Non ultimo, la riduzione delle dosi di carbamazepina tra il 2013 e il 2014 ha ragionevolmente contribuito a "sbloccare" l'attività della Vitamina D durante il recupero della funzione renale.

Come non ringraziare gli Autori per aver ricordato che la CKD-MBD merita sempre un approccio attento e globale! 


\section{Disclosures}

Financial support: No financial support was received for this submission.

Conflict of interest: Dr Galassi has received occasional speakers' fees from AbbVie and Shire.

\section{Andrea Galassi}

U.O. Nefrologia e Dialisi, A.O. Desio-Vimercate, Desio (MB)

Indirizzo per la corrispondenza:

Dr. Andrea Galassi

UO Nefrologia e Dialisi

AO Desio-Vimercate

Via Mazzini 1

20832 Desio (MB)

andrea.galassi@aovimercate.org

\section{Bibliografia}

1. Polidoro M. CKD-MBD: un caso "resistente". Giornale di Tecniche Nefrologiche e Dialitiche. 2014;26(4):348-53.

2. Dusso A, Arcidiacono MV. La carbamazepina antagonizza la soppressione di Vitamina D-mediata dell'iperparatiroidismo secondario: patogenesi e possibilità terapeutiche. Giornale di Tecniche Nefrologiche e Dialitiche. 2014;26(4):354-58.

3. Vezzoli G, Macrina L, Arcidiacono T. Citocromi P450 e interazione tra i farmaci. Giornale di Tecniche Nefrologiche e Dialitiche. 2014;26(4):359-60.

4. Kandula P, Dobre M, Schold JD, Schreiber MJ Jr, Mehrotra R, Navaneethan SD. Vitamin D supplementation in chronic kidney disease: a systematic review and meta-analysis of observational studies and randomized trials. Clin J Am Soc Nephrol. 2011;6(1):50-62.

5. Palmer SC, McGregor DO, Craig JC, Elder G, Macaskill $P$, Strippoli GF. Vitamin D compounds for people with chronic kidney disease requiring dialysis. Cochrane Database Syst Rev. 2009;(4):CD005633.

Published online: February 4, 2015 